\title{
Digital Detox
}

\author{
Milad Mirbabaie $\cdot$ Stefan Stieglitz $\cdot$ Julian Marx
}

Received: 26 November 2020 / Accepted: 21 December 2021 / Published online: 22 February 2022

(C) The Author(s) 2022

Keywords Digital detox · Technostress $\cdot$ Knowledge work $\cdot$ Information technology

\begin{abstract}
"We must learn to reawaken and keep ourselves awake, not by mechanical aids, but by an infinite expectation of the dawn, which does not forsake us even in our soundest sleep. I know of no more encouraging fact than the unquestionable ability of man to elevate his life by a conscious endeavour." — Henry David Thoreau, Walden
\end{abstract}

\section{Introduction}

The increasing use of information technology (IT) has a pervasive impact on society, including the world of work and its boundaries. Individual professionals, and knowledge workers in particular, are exposed to digital devices during the bulk of their working hours (Orlikowski and Scott 2016). In addition, persuasively designed social media and digital entertainment applications occupy the

Accepted after two revisions by Christine Legner.

\section{Mirbabaie ( $\square)$}

Faculty of Business Administration and Economics, Paderborn University, Warburger Str. 100, (Q3.128), 33098 Paderborn,

Germany

e-mail: milad.mirbabaie@uni-paderborn.de

\section{S. Stieglitz · J. Marx}

Department of Computer Science and Applied Cognitive

Science, Faculty of Engineering, Digital Communication and

Transformation, University of Duisburg-Essen, Forsthausweg 2, 47057 Duisburg, Germany leisure time of an unprecedented number of people. A recent study revealed that 33.1 million Germans use the Internet "multiple times a day", and 11 million even state to use it "constantly, almost the whole time" (Statista 2020). Scholarship clearly suggests that this compounded screen time can entail severe consequences to the wellbeing of individuals (Pflügner et al. 2020a). In fact, using IT can lead to technostress, which is defined as "any negative impact on attitudes, thoughts, behaviors, or body physiology that is caused either directly or indirectly by technology" (Weil and Rosen 1997, p. 5). Technostress constitutes a pressing social issue, especially with regards to changes in work-life boundaries, potentiated by the COVID-19 pandemic (Thomas et al. 2020). According to a study conducted in 2019, 86 percent of participants claimed that the inability to switch off devices after regular working hours has a negative effect on employee wellbeing (Stewart 2020). The result is a personal feeling of being overwhelmed by communication content and interpersonal online connections, which negatively affects work and private life alike (Gui and Büchi 2019).

To counteract technostress and its negative consequences on individual wellbeing and productivity, the notion of "digital detox" has found its way into popular culture and, more recently, Information Systems (IS) scholarship (Vaghefi et al. 2018; Eichner 2020; Zhou et al. 2020). Digital detox describes a periodic disconnection from IT as well as strategies which help to reduce the engagement with IT (Syvertsen and Enli 2019). Both its conceptualization and empirical analysis, however, have so far remained vague. Early research presents mixed results concerning the effectiveness of digital detox to improve individual wellbeing (Wilcockson et al. 2019; Brown and Kuss 2020; Schmuck 2020). Yet, making a statement about its effectiveness largely depends on the way digital detox is 
defined in each individual study. Despite this ambiguity, however, the literature commonly stresses the importance of remaining absent from IT for specified periods and calls for more research on this matter. The growing demand for digital detox before, during, and most likely after the COVID-19 pandemic fundamentally questions the way we use IT. Individuals increasingly find themselves yearning for time without the pervasive presence of IT (Fu et al. 2020). Digital detox, we argue, poses a symptom of a serious problem, that is, detrimental effects of IT use on health and work satisfaction. How can IS research help to get to the root of this problem?

\section{Digital Detox}

Digital detox describes a periodic disconnection from IT as well as strategies which help to reduce the engagement with IT (adapted from Syvertsen and Enli 2019)

In this article, we demonstrate the rationale behind digital detox and the developments in organizational knowledge work that precipitate the increasing popularity of periodically refraining from the use of IT. Moreover, we propose a first conceptualization of digital detox to guide future research in IS and beyond.

\section{Technostress in Organizations}

IT pervasively affects individuals' private and professional life (Tarafdar et al. 2019). Work arrangements built around steady IT use have become commonplace, in particular for knowledge workers (Kissmer et al. 2018). The latter are defined as workers whose occupation relies on "the creation, distribution or application of knowledge" (Davenport 2005, p. 9) Typical work arrangements allow knowledge workers to connect with people in geographical proximity, across greater distances, or completely remote-independently of time and space (Frick and Marx 2021). The COVID-19 pandemic has impelled a bulk of the workforce to switch to remote work arrangements, and home office respectively (Brynjolfsson et al. 2020). Whereas this development may be expected to be only temporary, leading firms such as Microsoft or Siemens have announced to preserve the ratio of remote work arrangements compared to regular office work beyond the pandemic (Newman 2020). This permanent shift means an empowerment of the knowledge worker in terms of her mobility and autonomy, while dismissing the paradigm of the corporate 9 to 5 job applied to knowledge work (Wang et al. 2020).
The flipside of the coin is that, with increasing IT use due to remote work arrangements, knowledge workers are exposed to a higher risk of technostress (Chandra et al. 2019). This phenomenon refers to stress individuals experience because of their IT use and their inability to cope with it healthily (Riedl et al. 2012; Mahapatra and Pillai 2018; Sarabadani et al. 2020). As employees often have to adapt to new and changing IT implemented by their organization, a number of scholars in IS focus on employee and IT professional related technostress (Chiu 2018; Mahapatra and Pillai 2018; Sarabadani et al. 2020).

\subsection{Theoretical Underpinnings of Technostress Literature}

Theoretically, the technostress literature heavily builds on the transactional model of stress (Lazarus and Folkman 1984). According to this model, individuals react cognitively to stimuli by assessing the motivational significance of a situation (primary appraisal). This may result in the perception of a situation to be irrelevant, benign-positive, or stressful. Subsequently, according to the model, individuals evaluate the assessment by contemplating possible actions to manage the situation (secondary appraisal). For example, one tries to find ways of alleviating possible harm in case of a stressful situation. The stressors perceived in the situation, in turn, provoke a stress reaction that can be of physiological, emotional, cognitive, or behavioral nature (strain). Finally, the individual may suffer from consequences caused by stress.

So far, IS literature has built on this model in a threefold manner. First, the transactional model has been tailored to technostress in organizations, defining the dimensions job characteristics, technological environment, organizational environment, and social environment, in which stressors can occur. Moreover, consequences of technostress do not only affect individual wellbeing, but may also impair performance, productivity, and IT user satisfaction (Adam et al. 2017). Second, specific techno-stressors have been defined, as shown in Table 1.

Third, technostress research has explored so-called coping strategies to mitigate technostress. With reference to the transactional perspective, these strategies can be problem-focused, e.g., stress-sensitive systems that provide live bio-feedback (Adam et al. 2017), or emotion-focused, e.g., mindfulness exercises (Pflügner et al. 2021). What the technostress literature has in common is that proposed coping strategies set in only after the second appraisal, i.e., after a stressor has been experienced and assessed as such. This constraint opens a new theoretical angle to approach technostress. 
Table 1 Techno-stressors according to Adam et al. (2017), Tarafdar et al. (2019), and Pflügner et al. (2021)

\begin{tabular}{ll}
\hline Techno-stressor & Description \\
\hline Techno-overload & Technology urging employees to work more and faster \\
Techno-invasion & Constant availability; blurring work/life boundaries \\
Techno-complexity & A perceived lack of abilities to meet the demands of IT use \\
Techno-insecurity & Fearing to lose one's job to IT or IT-savvy contenders \\
Techno-uncertainty & Uncertainty about changes in existing or new systems \\
Techno-unreliability & System malfunctions \\
\hline
\end{tabular}

\subsection{A Digital Detox Perspective on Technostress}

Research on technostress in organizations is guided by the assumption that individual exposure to techno-stressors is determined by job characteristics or technological, organizational, and social environments. Intervention through digital detox, we argue, can also start earlier, i.e., prior to the individual being exposed to the stimuli and technostressors, respectively. Figure 1 summarizes this supposed theoretical relationship.

If understood as a periodic disconnection and strategies to reduce the engagement with IT, digital detox has been investigated sporadically in technostress literature in the form of coping strategies. Under the label of 'behavioral disengagement', Hauk et al. (2019) describe the phenomenon of an individual "breaking off any further interaction and withdrawing from the stressful situation" (p. 22). Interestingly, the authors found this behavior to be counter-productive, with stress levels reducing in the shortterm, but then yoyoing back once the individual returns to the unresolved situation. Another study examined coping strategies which specifically answered to techno-invasion and techno-overload in organizations. Here, the authors proposed communication measures for employees and management that might be able to reduce the exposure to IT and its demands (Pflügner et al. 2020b). Strategies specifically aiming at the reduction of stimuli and successive stressors in the context of technostress in organizations, however, have not been part of the debate. Instead of changing the independent variable (IT exposure), research has focused on finding appropriate moderating forces (coping strategies) that alter the stress reactions and consequences. Digital detox, in this sense, offers an additional perspective (prevention strategies) that alleviates the predetermined experience of technostress when performing knowledge work.

\section{The Concept of Digital Detox}

Recently, the notion of digital detox has received increasing attention in academia, popular culture, and the self-help industry. The term 'detox' itself describes "a process or period of time in which one abstains from or rids the body

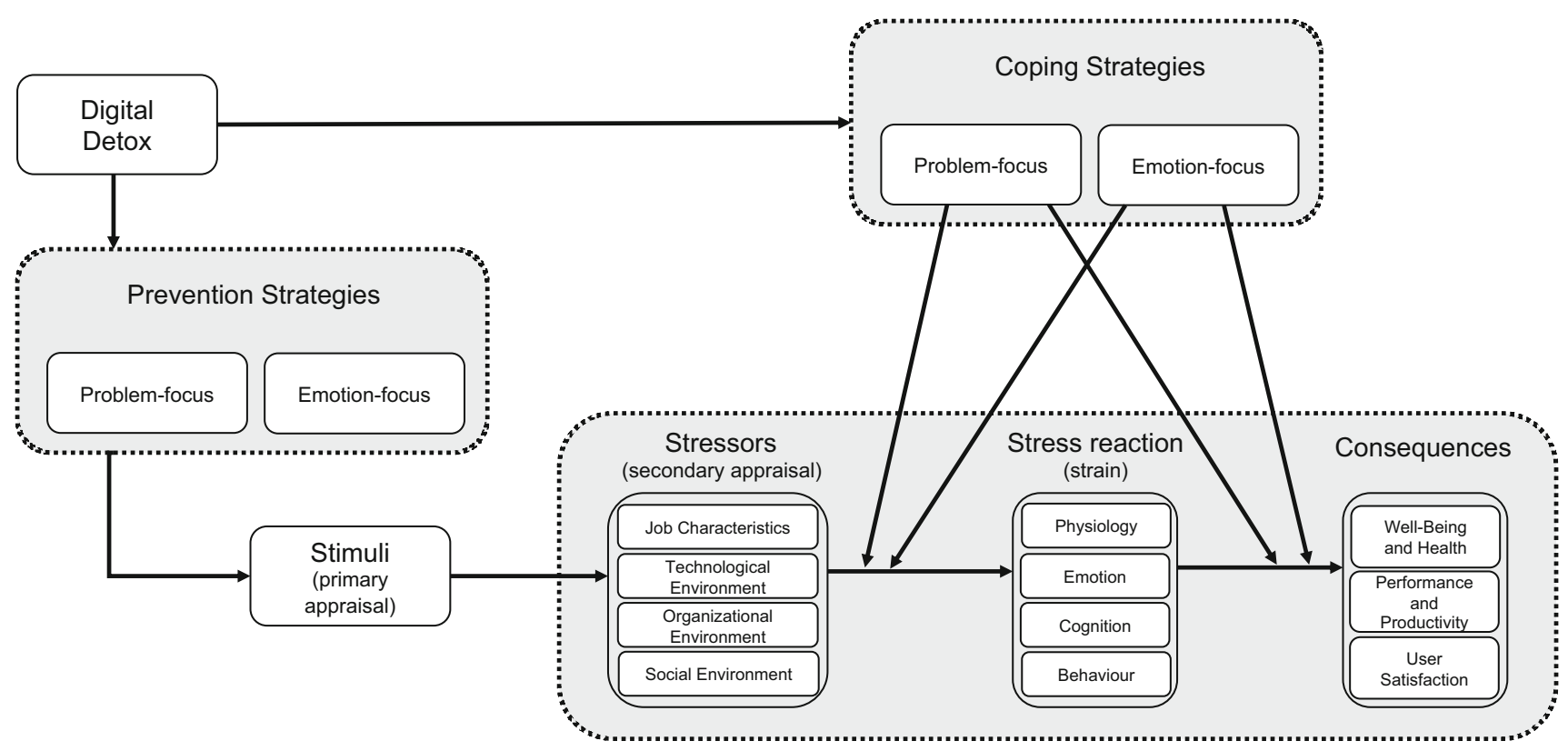

Fig. 1 The role of digital detox in the model of organizational technostress (adapted from Adam et al. 2016) 
of toxic or unhealthy substances" (Oxford Languages 2021). In medicine, the scientific grounding for detoxification is controversial - it can rather be seen as a consumer buzz word in conjunction with healthcare products (Cohen 2007). In the digital context, however, the effectiveness of detox measures is currently being scrutinized. Extant literature on digital detox goes back to around 2015 (Ugur and Koc 2015) and is dispersed across disciplines such as Psychology (Schmuck 2020), Media and Communication Studies (Syvertsen and Enli 2019), and IS (Mirbabaie et al. 2020).

\subsection{Digital Detox Research and Strategies}

So far, empirical studies report mixed results concerning the effectiveness of digital detox (Wilcockson et al. 2019; Brown and Kuss 2020; Schmuck 2020), while also stating the importance of more research on this matter. The ambiguity of empirical findings, however, is partly due to an inconsistent and often vague conceptualization of 'digital detox'. Moreover, viewed through a technostress lens, the phenomenon has been researched under the assumption of it being merely a coping strategy. This is reflected in recent definitions of digital detox, describing a process in which an individual abstains from objects that are perceived as unhealthy once exposure to them surpasses a certain point (Syvertsen and Enli 2019). Other terms like "digital diet" or "media diet" (Andersen et al. 2016) revolve around the same phenomenon as digital detox, which complicates consensus building. To establish the basis for a sound conceptualization of digital detox, Table 2 provides an overview of digital detox strategies that are prevalent in the literature.

For the purpose of this article, we refer to digital detox as an integrated approach to temporarily refrain from IT use to improve overall well-being and mental health. In doing so, we want to emphasize the preventive element of digital detox in addition to the coping element, as proposed by the transactional model of technostress (Adam et al. 2017). Henceforth, this catchword paper aims to shed light on the different strategies of digital detox and to establish the concept as a proper subsumption to the technostress literature.

\subsection{Toward a Conceptualization of Digital Detox in Organizational Contexts}

In the following, we aim to provide a first conceptualization of digital detox in organizations. As a first step, we take the identified digital detox strategies as shown in Table 1, and abstract three theoretical dimensions. First, we propose that the 'length of the interval' should be subject of scrutiny when researching digital detox. Second, we derive from existing strategies that they differ in the 'extent of intervention', that is in considering how much a digital detox strategy comes into conflict with organizational processes, norms, and behavior. Third, digital detox can have different 'levels of IT-assistance', e.g., through mindfulness apps, disabled e-mail servers after working hours, or calendar reminders to practice digital detox. Figure 2 provides three examples that range differently across the three dimensions of digital detox.

In this example, IT-free lunch breaks are minimal in length, intervention, and need for IT assistance. No-distraction appointments, on the other hand, can be medium in length and high in intervention and IT assistance. A mindfulness exercise may be chosen with short to medium length, no means of IT assistance and no interventions of organizational processes, norms, and behavior.

Below, we combine these dimensions with the transactional perspective known from the (organizational) technostress literature. Here, we consider both the motivational component of digital detox as well as the characteristics of a given digital detox strategy. Figure 3 depicts this integrated concept of digital detox.

This concept is useful for future research on digital detox for two reasons. First, digital detox proves to be a valuable concept to technostress and adjacent literature as it expands the theoretical chain of causation prevalent in current literature (stressors-strain-consequences followed by coping strategies to stressors-strain-consequences preceded by prevention strategies). Second, it allows for future research to determine a grading of digital detox strategies. This means that each measure (preventive or coping in nature) can be assessed and compared along the three dimensions.

\section{Research Agenda and Summary}

In conclusion, the interdependencies of knowledge work arrangements and technostress make a strong case for more research exploring the phenomenon of digital detox. Moreover, additional theorizing is necessary to understand, explain, and predict behavior related to digital detox. In this regard, it is imperative for IS research to examine how digital detox strategies can prevent technostress and what value this perspective adds to existing coping strategies (see Fig. 1). Possible research questions are:

- What are individual motivators for knowledge workers to conduct digital detox?

- How do individual digital detox strategies differ when motivated by prevention as opposed to coping?

- Which techno-stressors can be mitigated by preventive digital detox strategies? 
Table 2 Examples of digital detox strategies covered by literature

\begin{tabular}{|c|c|c|}
\hline Focus & Strategy & Supporting literature \\
\hline $\begin{array}{l}\text { Emotion-focused } \\
\text { strategies }\end{array}$ & Reflecting personal values and mindset & $\begin{array}{l}\text { Middleton and Cukier (2006); Syvertsen and Enli (2019); Pfaffinger } \\
\text { et al. (2020) }\end{array}$ \\
\hline \multirow[t]{2}{*}{ (individual) } & Emotion management & Al-Fudail and Mellar (2008) \\
\hline & Mindfulness training & Pflügner et al. (2021) \\
\hline $\begin{array}{l}\text { Problem-focused } \\
\text { strategies }\end{array}$ & Non-use, withdrawal, and time-outs & $\begin{array}{l}\text { Baumer et al. (2013); Braukmann et al. (2018); Mirbabaie et al. } \\
\text { (2020); Przybylski et al. (2021) }\end{array}$ \\
\hline \multirow[t]{6}{*}{ (individual) } & Self-regulation of usage behavior & $\begin{array}{l}\text { Uhls et al. (2014); Turel (2016); Anrijs et al. (2018); Stadin et al. } \\
\text { (2020) }\end{array}$ \\
\hline & Job transition & Butts et al. (2015) \\
\hline & Restricted social media use & Gui et al. (2017); Aranda and Baig (2018); Karmakar (2020) \\
\hline & Use of digital wellbeing applications & Gui et al. (2017); Eichner (2020); Karmakar (2020) \\
\hline & Switching to alternatives and offline behaviors & D’Arcy et al. (2014); Syvertsen and Enli (2019) \\
\hline & Segmentation of work and non-work & Sonnentag and Fritz (2015) \\
\hline $\begin{array}{l}\text { Problem-focused } \\
\text { strategies }\end{array}$ & $\begin{array}{l}\text { Top-down regulation of usage behavior (e.g. shut } \\
\text { down e-mail servers) }\end{array}$ & Görland and Kannengießer (2021) \\
\hline \multirow[t]{2}{*}{ (organizational) } & Organizational digital detox events & Karlsen (2020) \\
\hline & Training and support & Tarafdar et al. (2011); Pfaffinger et al. (2020) \\
\hline
\end{tabular}
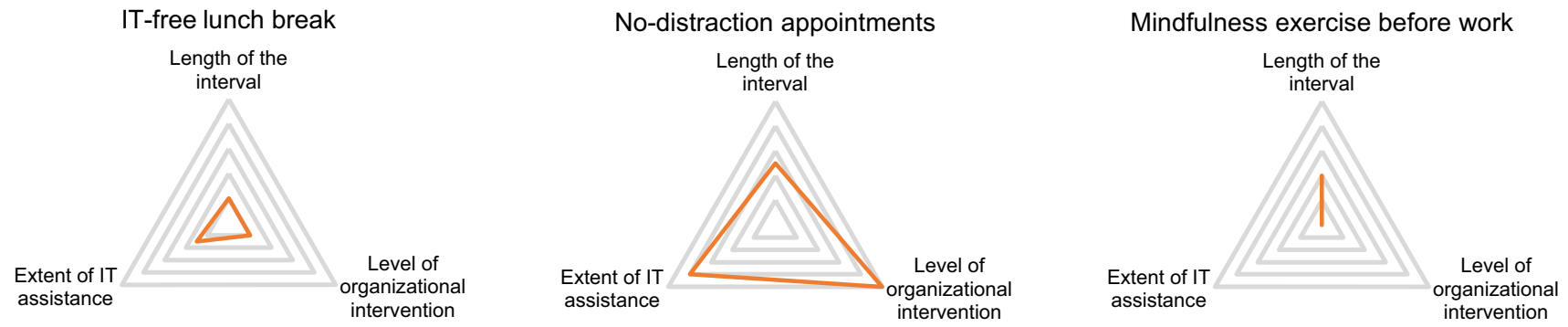

Fig. 2 Examples of digital detox strategies and their specification

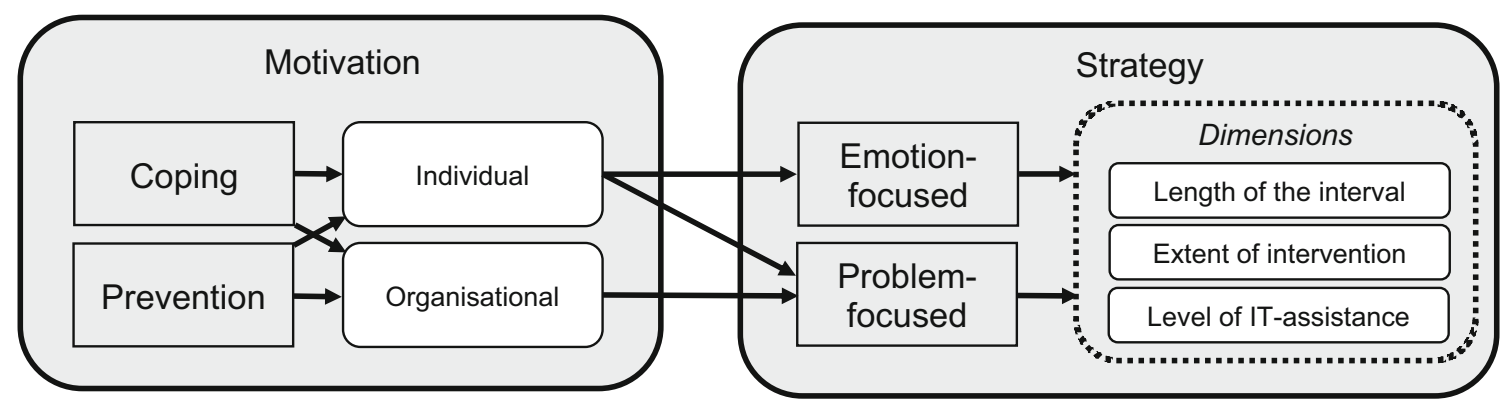

Fig. 3 Conceptualization of digital detox in organizations

Methodical approaches to these types of questions can vary, however, qualitative-interpretivist inquiries will help to obtain a phenomenological angle to digital detox. It is crucial to understand how individuals experience digital detox, what motivates them, and how it changes their work processes and technology use.

In addition, subsequent questions emerge when shifting to an organizational view on digital detox. As proposed by 
the transactional model of technostress, factors such as job characteristics, technological environment, organizational environment, and the social environment impact the exposure to techno-stressors (Adam et al. 2017). This line of argumentation yields further research questions:

- How do job characteristics affect the conduct of digital detox?

- How is the technological environment (number and types of devices, software, etc.) interrelated with digital detox?

- To what extent is management responsible for ensuring an organizational environment that appreciates digital detox?

- What are the implications of digital detox for the social environment inside organizations, e.g., in times of social isolation?

Here, hypothetico-deductive methods using quantitative data will help to test the theoretical relationships of digital detox, e.g., building on the transactional model of technostress.

In existing studies, due to conceptual ambiguity, the effectiveness of digital detox has been assessed with mixed results. Therefore, it will be important to clearly define what digital detox strategies are and how their impact can be measured. We call for research that systematically creates a taxonomy for digital detox strategies, e.g., by considering the proposed dimensions 'length of the interval', 'extent of intervention', and 'levels of IT-assistance'. Possible research questions in this regard are:

- What are digital detox strategies and how do they differ (e.g., from shortest and least interfering to longest and most interfering)?

- What are the implications of digital detox for IT design and the management of IT?

Finding answers to these questions will allow organizations, individual professionals, and IT designers to find a common ground that considers all interests at stake, that is, wellbeing, productivity, and user satisfaction.

In this catchword article, we proposed a first conceptualization of digital detox. We argue for digital detox as a phenomenon worthwhile to be examined as it adds a rather neglected perspective to technostress research. Instead of approaching technostress solely with coping mechanisms, digital detox offers a preventive and strategic element to technostress avoidance on both individual and organizational levels. We further stress the point that digital detox need not exclude IT dogmatically but should deploy it intelligently to support a user or organization to conscientiously implement digital detox strategies in their day-today operations. This will not only allow them to cope with technostress once a certain threshold is surpassed but helps to preventively regain equanimity and balance with regards to IT use. As Henry David Thoreau, the poet and philosopher we referenced in the opening quote to this article, would most certainly agree, digital detox may help us to elevate our lives by more conscious endeavors with and without IT.

Funding Open Access funding enabled and organized by Projekt DEAL.

Open Access This article is licensed under a Creative Commons Attribution 4.0 International License, which permits use, sharing, adaptation, distribution and reproduction in any medium or format, as long as you give appropriate credit to the original author(s) and the source, provide a link to the Creative Commons licence, and indicate if changes were made. The images or other third party material in this article are included in the article's Creative Commons licence, unless indicated otherwise in a credit line to the material. If material is not included in the article's Creative Commons licence and your intended use is not permitted by statutory regulation or exceeds the permitted use, you will need to obtain permission directly from the copyright holder. To view a copy of this licence, visit http://creativecommons. org/licenses/by/4.0/.

\section{References}

Adam MTP, Gimpel H, Maedche A, Riedl R (2017) Design blueprint for stress-sensitive adaptive enterprise systems. Bus Inf Syst Eng 59:277-291. https://doi.org/10.1007/s12599-016-0451-3

Al-Fudail M, Mellar H (2008) Investigating teacher stress when using technology. Comput Educ 51:1103-1110. https://doi.org/10. 1016/j.compedu.2007.11.004

Andersen K, de Vreese CH, Albæk E (2016) Measuring media diet in a high-choice environment - testing the list-frequency technique. Commun Methods Meas 10:81-98. https://doi.org/10.1080/ 19312458.2016.1150973

Anrijs S, Bombeke K, Durnez W, et al (2018) MobileDNA: relating physiological stress measurements to smartphone usage to assess the effect of a digital detox. In: Proceedings of the International Conference on Human-Computer Interaction, pp. 356-363

Aranda JH, Baig S (2018) Toward "JOMO": the joy of missing out and the freedom of disconnecting. In: Proceedings of the 20th International Conference on Human-Computer Interaction with Mobile Devices and Services, pp. 1-8

Baumer EPS, Adams P, Khovanskaya VD, et al. (2013) Limiting, leaving, and (re)lapsing: an exploration of Facebook non-use practices and experiences. In: Proceedings of the SIGCHI Conference on Human Factors in Computing Systems, pp. $3257-3266$

Braukmann J, Schmitt A, Ďuranová L, Ohly S (2018) Identifying ICT-related affective events across life domains and examining their unique relationships with employee recovery. J Bus Psychol 33:529-544. https://doi.org/10.1007/s10869-017-9508-7

Brown L, Kuss DJ (2020) Fear of missing out, mental wellbeing, and social connectedness: a seven-day social media abstinence trial. Int J Environ Res Public Health 17:1-18. https://doi.org/10.3390/ ijerph17124566

Brynjolfsson E, Horton JJ, Ozimek A et al. (2020) Covid-19 and remote work: an early look at US data. No. w27344. National 
Bureau of Economic Research. Doi: https://doi.org/10.3386/ w27344

Butts MM, Becker WJ, Boswell WR (2015) Hot buttons and time sinks: the effects of electronic communication during nonwork time on emotions and work-nonwork conflict. Acad Manag J 58:763-788. https://doi.org/10.5465/amj.2014.0170

Chandra S, Shirish A, Srivastava SC (2019) Does technostress inhibit employee innovation? Examining the linear and curvilinear influence of technostress creators. Commun Assoc Inf Syst 44:299-331. https://doi.org/10.17705/1CAIS.04419

Chiu CM (2018) Impacts of technostress creators and personal resources on IT professionals' strain and engagement. In: Proceedings of the 22nd Pacific Asia Conference on Information Systems, pp. 1-7

Cohen M (2007) "Detox": science or sales pitch? Aust Fam Physician 36:1009-1010

D'Arcy J, Gupta A, Tarafdar M, Turel O (2014) Reflecting on the "dark side" of information technology use. Commun Assoc Inf Syst 35:109-118. https://doi.org/10.17705/1cais.03505

Davenport TH (2005) Thinking for a living: how to get better performance and results from knowledge workers. Harvard Business School Publishing, Boston

Eichner AA (2020) Planting trees and tracking screen time: a taxonomy of digital wellbeing features. In: Proceedings of the 24th Pacific Asia Conference on Information Systems, pp. 1-14

Frick N, Marx J (2021) Integrating digital nomads in corporate structures: managerial contemplations. In: Proceedings of the 54th Hawaii International Conference on System Sciences, pp. 2111-2120

Fu S, Li H, Liu Y et al (2020) Social media overload, exhaustion, and use discontinuance: examining the effects of information overload, system feature overload, and social overload. Inf Process Manag 57:102307. https://doi.org/10.1016/j.ipm.2020.102307

Görland SO, Kannengießer S (2021) A matter of time? Sustainability and digital media use. Digit Policy Regul Gov 23:248-261. https://doi.org/10.1108/DPRG-11-2020-0160

Gui M, Büchi M (2019) From use to overuse: digital inequality in the age of communication abundance. Soc Sci Comput Rev. https:// doi.org/10.1177/0894439319851163

Gui M, Fasoli M, Carradore R (2017) Digital well-being. Developing a new theoretical tool for media literacy research. Ital J Sociol Educ 9:155-173. https://doi.org/10.14658/pupj-ijse-2017-1-8

Hauk N, Göritz AS, Krumm S (2019) The mediating role of coping behavior on the age-technostress relationship: a longitudinal multilevel mediation model. PLoS ONE 14:1-22. https://doi.org/ 10.1371/journal.pone.0213349

Karlsen F (2020) Digital detox camp: values and motivations for engaging in digital disconnect. In: The 21st Annual Conference of the Association of Internet Researchers, pp. 1-4

Karmakar I (2020) A review of IT addiction in IS research. In: Proceedings of the 26th American Conference on Information Systems

Kissmer T, Knoll J, Stieglitz S, Gross R (2018) Knowledge workers' expectations towards a digital workplace. In: Proceeedings of the 24th Americas Conference on Information Systems, pp. 1-10

Oxford Languages (2021) Detox. Oxford Lang

Lazarus RS, Folkman S (1984) Stress, appraisal, and coping. Springer, New York

Mahapatra M, Pillai R (2018) Techonostress in organizations: a review of literature. In: Proceedings of the 26th European Conference on Information Systems, pp. 1-15

Middleton CA, Cukier W (2006) Is mobile email functional or dysfunctional? Two perspectives on mobile email usage. Eur J Inf Syst 15:252-260. https://doi.org/10.1057/palgrave.ejis. 3000614
Mirbabaie M, Marx J, Braun L-M, Stieglitz S (2020) Digital detox mitigating digital overuse in times of remote work and social isolation. In: Proceedings of the 31st Australasian Conference on Information Systems, pp. 1-8

Newman D (2020) Distributed companies, work from home, and the technology enabling the change. Forbes

Orlikowski WJ, Scott SV (2016) Digital work: a research agenda. In: Czarniawska B (ed) A Research Agenda for Management and Organization Studies. Elgar, Cheltenham, pp 88-95

Pfaffinger KF, Reif JAM, Spie $\beta$ E (2020) When and why telepressure and technostress creators impair employee well-being. Int $\mathrm{J}$ Occup Saf Ergon. https://doi.org/10.1080/10803548.2020. 1846376

Pflügner K, Maier C, Mattke J, Weitzel T (2020a) Personality profiles that put users at risk of perceiving technostress. Bus Inf Syst Eng 63(4):389-402. https://doi.org/10.1007/s12599-020-00668-7

Pflügner K, Maier C, Weitzel T (2021) The direct and indirect influence of mindfulness on techno-stressors and job burnout: a quantitative study of white-collar workers. Comput Hum Behav 115:106566. https://doi.org/10.1016/j.chb.2020.106566

Pflügner K, Reis L, Maier C, Weitzel T (2020b) Communication measures to reduce techno-invasion and techno-overload: a qualitative study uncovering positive and adverse effects. In: Proceedings of the Conference on Computers and People Research, pp. 114-122. Doi: https://doi.org/10.1145/3378539. 3393855

Przybylski AK, Nguyen TT, Law W, Weinstein N (2021) Does taking a short break from social media have a positive effect on wellbeing? Evidence from three preregistered field experiments. J Technol Behav Sci 6:507-514. https://doi.org/10.1007/s41347020-00189-w

Riedl R, Kindermann H, Auinger A, Javor A (2012) Technostress from a neurobiological perspective: system breakdown increases the stress hormone cortisol in computer users. Bus Inf Syst Eng 4:61-69. https://doi.org/10.1007/s12599-012-0207-7

Sarabadani J, Compeau D, Carter M (2020) An investigation of IT users' emotional responses to technostress creators. In: Proceedings of the 53rd Hawaii International Conference on System Sciences, pp. 6113-6122

Schmuck D (2020) Does digital detox work? Exploring the role of digital detox applications for problematic smartphone use and well-being of young adults using multigroup analysis. Cyberpsychol Behav Soc Netw 23:526-532. https://doi.org/10.1089/cyber. 2019.0578

Sonnentag S, Fritz C (2015) Recovery from job stress: the stressordetachment model as an integrative framework. J Organ Behav 36:73-102. https://doi.org/10.1002/job

Stadin M, Nordin M, Fransson EI, Broström A (2020) Healthcare managers' experiences of technostress and the actions they take to handle it - a critical incident analysis. BMC Med Inform Decis Mak 20:1-11. https://doi.org/10.1186/s12911-020-01261-

Statista (2020) Statistics on internet use in Germany. https://de. statista.com/themen/2033/internetnutzung-in-deutschland/. Accessed 26 January 2022

Stewart C (2020) Negative effects of technological advances on the well-being of employees within organizations in the United Kingdom (UK) in 2019. https://www.statista.com/statistics/ 1134262/negative-effect-of-tech-on-employee-s-well-being-inthe-uk/. Accessed 26 January 2022

Syvertsen T, Enli G (2019) Digital detox: media resistance and the promise of authenticity. Converg Int J Res into New Media Technol. https://doi.org/10.1177/1354856519847325

Tarafdar M, Tu Q, Ragu-Nathan TS, Ragu-Nathan BS (2011) Crossing to the dark side: examining creators, outcomes, and 
inhibitors of technostress. Commun ACM 54:113-120. https:// doi.org/10.1145/1995376.1995403

Tarafdar M, Maier C, Laumer S, Weitzel T (2019) Explaining the link between technostress and technology addiction for social networking sites: a study of distraction as a coping behavior. Inf Syst J 30:96-124. https://doi.org/10.1111/isj.12253

Thomas O, Hagen S, Frank U et al (2020) Global crises and the role of BISE. Bus Inf Syst Eng 62:385-396. https://doi.org/10.1007/ s12599-020-00657-w

Turel $\mathrm{O}$ (2016) Untangling the complex role of guilt in rational decisions to discontinue the use of a hedonic Information System. Eur J Inf Syst 25:432-447. https://doi.org/10.1057/ s41303-016-0002-5

Ugur NG, Koc T (2015) Time for digital detox: misuse of mobile technology and phubbing. Procedia - Soc Behav Sci 195:1022-1031. https://doi.org/10.1016/j.sbspro.2015.06.491

Uhls YT, Michikyan M, Morris J et al (2014) Five days at outdoor education camp without screens improves preteen skills with nonverbal emotion cues. Comput Hum Behav 39:387-392. https://doi.org/10.1016/j.chb.2014.05.036

Vaghefi I, Xiao B, Turel O, Matta V (2018) Digital detox? Understanding users' abstinence from social networking sites use. In: Proceedings of the 39th International Conference on Information Systems, pp. 1-4

Wang B, Schlagwein D, Cecez-Kecmanovic D, Cahalane MC (2020) Beyond the factory paradigm: digital nomadism and the digital future(s) of knowledge work post-COVID-19. J Assoc Inf Syst 21:1379-1401. https://doi.org/10.17705/1jais.00641

Wilcockson TDW, Osborne AM, Ellis DA (2019) Digital detox: the effect of smartphone abstinence on mood, anxiety, and craving. Addict Behav 99:2-5. https://doi.org/10.1016/j.addbeh.2019.06. 002

Zhou Z, Li X, Li X (2020) Conceptualizing restricted use in the social media context. In: Proceeedings of the 26th Americas Conference on Information Systems, pp. 1-5 